\title{
CONVERGENCE RESULTS FOR MHD SYSTEM
}

\author{
RIDHA SELMI
}

Received 14 April 2005; Revised 29 September 2005; Accepted 26 January 2006

Dedicated to Mouna

A magnetohydrodynamic system is investigated in both cases of the periodic domain $\mathbb{T}^{3}$ and the whole space $\mathbb{R}^{3}$. Existence and uniqueness of strong solution are proved. Asymptotic behavior of the solution when the Rossby number $\varepsilon$ goes to zero is studied. The proofs use the spectral properties of the penalization operator and involve Friedrich's method, Schochet's methods, and product laws in Sobolev spaces of sufficiently large exponents.

Copyright (c) 2006 Hindawi Publishing Corporation. All rights reserved.

\section{Introduction and summary of results}

In this paper, we study the existence, the uniqueness, and the asymptotic behavior of strong solutions of the following MHD system for a 3D incompressible ideal fluid:

$$
\begin{gathered}
\partial_{t} u-\varepsilon \Delta u+u \cdot \nabla u-\operatorname{curl} b \times b+\frac{1}{\varepsilon} \operatorname{curl} b \times e_{2}+\frac{1}{\varepsilon} u \times e_{3}=-\nabla p \quad \text { in } \mathbb{R}^{+} \times \Omega, \\
\partial_{t} b-\frac{1}{\varepsilon} \Delta b+u \cdot \nabla b-b \cdot \nabla u+\frac{1}{\varepsilon} \operatorname{curl}\left(u \times e_{2}\right)=0 \quad \text { in } \mathbb{R}^{+} \times \Omega, \\
\operatorname{div} u=0 \quad \text { in } \mathbb{R}^{+} \times \Omega, \\
\operatorname{div} b=0 \quad \text { in } \mathbb{R}^{+} \times \Omega,
\end{gathered}
$$

where the velocity field $u$, the induced magnetic perturbation $b$, and the pressure $p$ are unknown functions of time $t$ and space variables $x=\left(x_{1}, x_{2}, x_{3}\right), e_{2}$ and $e_{3}$ are, respectively, the second and the third vectors of the Cartesian coordinate system, $\Omega$ is either the torus $\mathbb{T}^{3}$ or the whole space $\mathbb{R}^{3}$ and $\varepsilon$ is a small positive parameter destined to go to zero. The above system is a particular modelization of the MHD flow in the earth's core which is believed to support a self-excited dynamo process generating the earth's magnetic field. In a more general case, throughout their paper [7], Desjardins et al. have modelized this 
process by the following general formulation:

$$
\begin{gathered}
\partial_{t} u+u \cdot \nabla u+\frac{\nabla p}{\varepsilon}-\frac{E}{\varepsilon} \Delta u+\frac{e \times u}{\varepsilon}=\frac{\Lambda}{\varepsilon} \operatorname{curl} b \times e^{\prime}+\frac{\Lambda \theta}{\varepsilon} \operatorname{curl} b \times b \quad \text { in } \mathbb{R}^{+} \times \Omega, \\
\partial_{t} b+u \cdot \nabla b=b \cdot \nabla u+\frac{\operatorname{curl}\left(u \times e^{\prime}\right)}{\theta}+\frac{\Delta b}{\theta} \quad \text { in } \mathbb{R}^{+} \times \Omega, \\
\operatorname{div} u=0 \quad \text { in } \mathbb{R}^{+} \times \Omega, \quad \operatorname{div} b=0 \quad \text { in } \mathbb{R}^{+} \times \Omega,
\end{gathered}
$$

where $E, \varepsilon, \Lambda$, and $\theta$ represent, respectively, the Ekman number, the Rossby number, the Elsasser number and the magnetic Reynolds number, $e$ is a fixed vector axe of rotation of the earth, and $e^{\prime}$ is the time-independent component of the earth's magnetic field $B$ defined in [7] by

$$
B=e^{\prime}+\theta b
$$

Here we take $E=\varepsilon^{2}, \Lambda=1, \theta=\varepsilon, e=-e_{3}$, and $e^{\prime}=-e_{2}$. We believe that our choice belongs to the parameter range relevant to the earth's core as it satisfies the following ordering (see $[7,8])$ :

$$
\varepsilon \longrightarrow 0, \quad \Lambda=\mathbb{O}(1), \quad \varepsilon \theta \longrightarrow 0, \quad E \sim \varepsilon^{2} .
$$

Physically, according to [8], letting the Rossby number $\varepsilon$ go to zero and consequently the Ekman number means that inertial forces and viscous attraction-repulsion modelized, respectively, by $\partial_{t} u+u \cdot \nabla u$ and $\varepsilon \Delta u$ are of neglected effects compared to the Coriolis one due to rotation of the earth in the direction $e_{3}$ with the high speed $1 / \varepsilon$. The aim of this study is to find the limit system, when $\varepsilon$ goes to zero, which physically corresponds to the equations characterizing the magnetostrophic equilibrium. For more physical details, we refer the readers to $[7,8]$.

We denote by $\mathbb{P}$ the $L^{2}$ orthogonal projection on divergence-free vector fields. Applying $\mathbb{P}$ to $(1.1)$, one can see that $U:=(u, b)$ is a solution of the following abstract system:

$$
\begin{gathered}
\partial_{t} U+Q(U, U)+a_{2}^{\varepsilon}(D) U+L^{\varepsilon}(U)=0 \quad \text { in } \mathbb{R}^{+} \times \Omega, \\
\operatorname{div} u=\operatorname{div} b=0,
\end{gathered}
$$

where, if we set $U=(u, b)$, the quadratic term $Q$ is defined by

$$
Q(U, U)=(\mathbb{P}(u \cdot \nabla u)-\mathbb{P}(\operatorname{curl} b \times b), u \cdot \nabla b-b \cdot \nabla u),
$$

the viscous term is

$$
a_{2}^{\varepsilon}(D) U=\left(-\varepsilon \Delta u,-\frac{1}{\varepsilon} \Delta b\right)
$$

and the linear perturbation $L^{\varepsilon}$ is given by

$$
L^{\varepsilon}(U)=\frac{1}{\varepsilon} L(U):=\frac{1}{\varepsilon}\left(\mathbb{P}\left(u \times e_{3}\right)+\mathbb{P}\left(\operatorname{curl}(b) \times e_{2}\right), \operatorname{curl}\left(u \times e_{2}\right)\right) .
$$


We note that $Q(U, V)=(A U \cdot \nabla V+A V \cdot \nabla U) / 2$, where $A$ is a linear operator, $a_{2}^{\varepsilon}(D)$ is elliptic, and $L^{\varepsilon}=(1 / \varepsilon) L$ is a skew-symmetric linear operator. This skew-symmetry is an important property for the existence result since the perturbation disappears in the energy estimate.

Singular limits in systems such as $\left(\mathscr{S}^{\varepsilon}\right)$ have been studied by several authors. In the hyperbolic case, Babin et al. [1] studied the incompressible rotating Euler equation on the torus. Using the method introduced by Schochet in $[18,19]$, Gallagher studied, respectively, in $[9,10]$ this problem in its abstract parabolic and hyperbolic form. In the case of the incompressible rotating Navier-Stokes equation on the torus, it is shown in $[1,11]$ that the solutions converge to a solution of a certain diffusion equation. Moreover, for a special initial condition, there exists a sequence of solutions convergent to a solution of a two-dimensional Navier-Stokes equation. MHD systems were investigated, respectively, with the choice of parameters $E=\varepsilon^{2}, \Lambda=1, \theta=\varepsilon$, and $e=e^{\prime}=-e_{3}$ in [2] and $E=\varepsilon^{2}$, $\Lambda=\varepsilon, \theta=1$, and $e=e^{\prime}=-e_{3}$ in [3]. We also refer to the results proved in $[6,12-14,16]$.

We specify in this paper to choose $e^{\prime}$ to be equal to $-e_{2}$. Physically, this choice deals with the effect of the longitudinal component of the time-independent magnetic field $B$ on the dynamo process. The mathematical study in this case requires to use in a precise way the structure and the spectral properties of the penalization operator to establish the convergence results. Such structure, namely, the $e_{2}$ dependence, allows us to put the system in the appropriate form before applying Schochet's method.

We begin by establishing an existence result which follows directly from the Friedrichs method and the energy estimate. Precisely, we will prove in Section 2 local existence of strong solutions on uniform time, namely, solutions given in the following theorem.

Theorem 1.1. Let $s>3 / 2+2$ be an integer and $U_{0}=\left(u_{0}, b_{0}\right) \in H^{s}(\Omega)$ such that div $u_{0}=$ $\operatorname{div} b_{0}=0$. Then, there exist $T>0$ and a constant $C>0$ such that, for all $\varepsilon>0$, there exists $a$ unique solution $U^{\varepsilon} \in \mathscr{C}^{0}\left([0, T], H^{s}(\Omega)\right) \cap L^{2}\left([0, T], H^{s+1}(\Omega)\right)$ of the system $\left(\mathscr{C}^{\varepsilon}\right)$ satisfying, for all $t \in[0, T]$,

$$
\left\|U^{\varepsilon}(t)\right\|_{H^{s}(\Omega)}^{2}+2 \varepsilon \int_{0}^{t}\left\|\nabla u^{\varepsilon}\right\|_{H^{s}(\Omega)}^{2}+\frac{2}{\varepsilon} \int_{0}^{t}\left\|\nabla b^{\varepsilon}\right\|_{H^{s}(\Omega)}^{2} \leq 5\left\|U_{0}\right\|_{H^{s}(\Omega)}^{2} .
$$

Moreover, if $\left\|\left(u_{0}, b_{0}\right)\right\|_{H^{s}(\Omega)} \leq c \mathcal{E}$, then the solution $U^{\varepsilon}$ is global.

Once the existence result is established, we turn to the asymptotic behavior of the strong solution of $\left(\mathscr{S}^{\varepsilon}\right)$ when $\varepsilon$ goes to zero. Since $\partial_{t} U^{\varepsilon}$ is not a priori bounded in $\varepsilon$, the classical proofs used, for example, in $[15,20]$ and based on taking the limit directly in the system no longer work.

In the case of the torus $\mathbb{T}^{3}$, this difficulty will be avoided by writing the velocity field $u^{\varepsilon}$ in the following form:

$$
u^{\varepsilon}=u_{\mathrm{osc}}^{\varepsilon}+\overline{u^{\varepsilon}}
$$

where

$$
\overline{u^{\varepsilon}}\left(t, x_{1}, x_{3}\right)=\int_{\mathbb{T}} u^{\varepsilon}\left(t, x_{1}, x_{2}, x_{3}\right) d x_{2}
$$


The asymptotic behavior of each term of $u^{\varepsilon}$ will be investigated separately. For the oscillating part $u_{\mathrm{osc}}^{\varepsilon}$, spectral properties of $L^{\varepsilon}$ and Lebesgue's convergence theorem allow to conclude in the case of low frequencies. For high frequencies, the nonlinear part will be investigated term by term; the convergence result is due to the energy estimate and the smallness of the nonlinearity. To study the nonoscillating component $\overline{u^{\varepsilon}}$, we average the first MHD equation in the second space variable to obtain a two-dimensional Navier-Stokes equation singularly perturbed by the well-known linear operator $L(u):=\mathbb{P}\left(u \times e_{3}\right)$. Following the method introduced by Schochet in [19], we filter the system by the associated group $\mathscr{L}(t)$ in order to look for the limit system (in the sense of distributions) satisfied by the possible limit $v$ of the filtered solution $v^{\varepsilon}:=\mathscr{L}(-t / \mathcal{\varepsilon}) \overline{u^{\varepsilon}}$.

For the induction equation, we prove that the diffusion process vanishes as the Rossby number goes to zero. The magnetic perturbation $b^{\varepsilon}$ conserves its initial average on the torus, but its oscillating component $b_{\mathrm{osc}}^{\varepsilon}$ has the same behavior as $u_{\mathrm{osc}}^{\varepsilon}$. More precisely, we prove the following convergence results.

Theorem 1.2. Let $s>3 / 2+2$ be an integer and $U_{0}=\left(u_{0}, b_{0}\right) \in H^{s}\left(\mathbb{T}^{3}\right)$ such that div $u_{0}=$ $\operatorname{div} b_{0}=0$. Let $U^{\varepsilon}=\left(u^{\varepsilon}, b^{\varepsilon}\right)$ be the family of solutions of $\left(\mathscr{G}^{\varepsilon}\right)$ given by Theorem 1.1. Then

$$
\begin{gathered}
\nabla b^{\varepsilon} \longrightarrow 0 \quad \text { in } L^{2}\left([0, T], H^{s}\left(\mathbb{T}^{3}\right)\right), \\
\int_{\mathbb{T}^{3}} b^{\varepsilon}(t, x) d x=\int_{\mathbb{T}^{3}} b_{0}(x) d x \quad \forall t \in[0, T], \\
\left.\left.\left(u_{\mathrm{osc}}^{\varepsilon}, b_{\mathrm{osc}}^{\varepsilon}\right) \longrightarrow(0,0) \quad \text { in } L_{\mathrm{loc}}^{\infty}(] 0, T\right], H^{s-1}\left(\mathbb{T}^{3}\right)\right) .
\end{gathered}
$$

Moreover, for all $s^{\prime}<s$, the family $v^{\varepsilon}:=\mathscr{L}(-t / \varepsilon) \overline{u^{\varepsilon}}$ converges strongly in $\mathscr{C}^{0}\left([0, T], H^{s^{\prime}}\left(\mathbb{T}^{3}\right)\right)$ to the solution $v$ of the following two-dimensional limit system:

$$
\begin{gathered}
\partial_{t} v+Q^{0}(v, v)=0 \quad \text { in }[0, T] \times \mathbb{T}^{2}, \\
\partial_{1} v_{1}+\partial_{3} v_{3}=0, \\
\left.v\right|_{t=0}=\overline{u_{0}},
\end{gathered}
$$

where, $Q^{0}(v, v):=\lim _{\mathcal{\varepsilon} \rightarrow 0} \mathscr{L}(-t / \mathcal{E}) \mathbb{P}(\mathscr{L}(t / \varepsilon) v \cdot \nabla \mathscr{L}(t / \varepsilon) v)$, in the sense of distributions.

Theorem 1.2 deserves a few comments that will be summarized in the following remark.

Remark 1.3. (1) Following the notation of Theorem 1.2, we note that $\mathscr{L}(t / \varepsilon) v^{\varepsilon}$ is the noncompact part of $u^{\varepsilon}$.

(2) Results obtained about the magnetic perturbation are in accordance with physicist's suggestions. In fact, according to [8], the creation and diffusion of the earth's magnetic field needed by a self-exited dynamo process necessitates a Reynolds number greater than the unity.

(3) An explicit computation of the resonance set of the limit quadratic term $Q^{0}(v, v)$ seems not to be an easy matter. For this reason, we will just give a particular example to show that this set is not empty and then the considered torus is a resonant one. 
In the case of the whole space $\mathbb{R}^{3}$, we mention that the plane $\left\{\xi \in \mathbb{R}^{3}, \xi_{2}=0\right\}$, where the resonance phenomenon is supposed to take place, is zero Lebesgue measure. That is why the following theorem holds.

Theorem 1.4. Let $s>3 / 2+2$ be an integer and $U_{0}=\left(u_{0}, b_{0}\right) \in H^{s}\left(\mathbb{R}^{3}\right)$ such that div $u_{0}=$ $\operatorname{div} b_{0}=0$. Let $U^{\varepsilon}=\left(u^{\varepsilon}, b^{\varepsilon}\right)$ be the family of solutions of $\left(\varphi^{\varepsilon}\right)$ given by Theorem 1.1. Then

$$
\begin{gathered}
b^{\varepsilon} \longrightarrow 0 \quad \text { in } L_{T}^{2}\left(\dot{H}^{s+1}\left(\mathbb{R}^{3}\right)\right) \\
\left.\left.\left(u^{\varepsilon}, b^{\varepsilon}\right) \longrightarrow(0,0) \quad \text { in } L_{\mathrm{loc}}^{\infty}(] 0, T\right], H^{s-1}\left(\mathbb{R}^{3}\right)\right) .
\end{gathered}
$$

This paper is organized as follow. In the next section, we present the proof of the existence result (Theorem 1.1). Section 3 is devoted to the proof of the convergence results on the torus (Theorem 1.2). Finally, we turn to the convergence results in the case of the whole space ( Theorem 1.4).

\section{Existence result}

In this section, we prove Theorem 1.1. Notice that this proof is similar to that of $[2,3$, Theorem 1.2]. We include it here for the convenience of the reader.

We begin by observing that by classical energy methods one can prove global existence of the so-called "Leray's solutions" for the system $\left(\mathscr{Y}^{\varepsilon}\right)$ and derive the following $L^{2}$-energy estimate:

$$
\left\|U^{\varepsilon}(t)\right\|_{L^{2}(\Omega)}^{2}+2 \varepsilon \int_{0}^{t}\left\|\nabla u^{\varepsilon}(\tau)\right\|_{L^{2}(\Omega)}^{2} d \tau+\frac{2}{\varepsilon} \int_{0}^{t}\left\|\nabla b^{\varepsilon}(\tau)\right\|_{L^{2}(\Omega)}^{2} d \tau \leq\left\|U_{0}\right\|_{L^{2}(\Omega)}^{2} .
$$

To study the existence and the regularity of strong solutions, we first approximate the nonlinear part of MHD system by a family of nicer nonlinearities, for which we can apply the classical theory of ODE in order to construct approximate solutions as in [4], for example. Next, we obtain uniform estimates on the approximate solutions, by using the conservation laws. Finally, we use these estimates to pass to the limit in the approximate equation. Precisely, we introduce, for a strictly positive integer $n$, the Friedrichs operator $J_{n}$ defined by

$$
J_{n} u=\mathscr{F}^{-1}\left(\mathbf{1}_{B(0, n)} \hat{u}\right) .
$$

We consider the following approximate magnetohydrodynamic system $\left(\mathrm{MHD}_{n}\right)$ :

$$
\begin{gathered}
\partial_{t} u_{n}-\varepsilon \Delta J_{n} u_{n}+J_{n} \operatorname{div}\left(J_{n} u_{n} \otimes J_{n} u_{n}\right)-J_{n} \operatorname{div}\left(J_{n} b_{n} \otimes J_{n} b_{n}\right)+\frac{1}{\varepsilon} \partial_{2}\left(J_{n} b_{n}\right)+\frac{J_{n} u_{n} \times e_{3}}{\varepsilon} \\
=\nabla \Delta^{-1} \operatorname{div}\left(J_{n} \operatorname{div}\left(J_{n} u_{n} \otimes J_{n} u_{n}\right)-J_{n} \operatorname{div}\left(J_{n} b_{n} \otimes J_{n} b_{n}\right)+\frac{J_{n} u_{n} \times e_{3}}{\varepsilon}\right), \\
\partial_{t} b_{n}-\frac{1}{\varepsilon} \Delta J_{n} b_{n}+J_{n} \operatorname{div}\left(J_{n} u_{n} \otimes J_{n} b_{n}\right)-J_{n} \operatorname{div}\left(J_{n} b_{n} \otimes J_{n} u_{n}\right)+\frac{1}{\varepsilon} \partial_{2}\left(J_{n} u_{n}\right)=0, \\
\left(\left.u_{n}\right|_{t=0},\left.b_{n}\right|_{t=0}\right)=\left(J_{n} u_{0}, J_{n} b_{0}\right) .
\end{gathered}
$$


The above system is an ODE and can be written in the following abstract form:

$$
\partial_{t} U_{n}=F_{n}\left(U_{n}\right)
$$

where $U_{n}=\left(u_{n}, b_{n}\right)$ and the expression of $F_{n}$ is given by the $\left(\mathrm{MHD}_{n}\right)$ system. Since $F_{n}$ is a continuous function from $H^{\sigma}(\Omega)$ into $H^{\sigma}(\Omega)$ for all $\sigma \in \mathbb{R}$, then $\left(\mathrm{MHD}_{n}\right)$ has a unique maximal solution $U_{n}$ in the space $\mathscr{C}^{1}\left(\left[0, T_{n}^{*}(\varepsilon)\left[, H^{s}(\Omega)\right)\right.\right.$. Since $J_{n}^{2}=J_{n}$ and $\operatorname{div} u_{n}$ $=\operatorname{div} b_{n}=0$, it follows by uniqueness that

$$
\begin{gathered}
\partial_{t} u_{n}-\varepsilon \Delta u_{n}+J_{n}\left(u_{n} \cdot \nabla u_{n}\right)-J_{n}\left(b_{n} \cdot \nabla b_{n}\right)+\frac{1}{\varepsilon} \partial_{2} b_{n}+\frac{1}{\varepsilon} u_{n} \times e_{3} \\
=\nabla \Delta^{-1} \operatorname{div}\left(J_{n} \operatorname{div}\left(u_{n} \otimes u_{n}\right)-J_{n} \operatorname{div}\left(b_{n} \otimes b_{n}\right)+\frac{u_{n} \times e_{3}}{\varepsilon}\right), \\
\partial_{t} b_{n}-\frac{1}{\varepsilon} \Delta b_{n}+J_{n}\left(u_{n} \cdot \nabla b_{n}\right)-J_{n}\left(b_{n} \cdot \nabla u_{n}\right)+\frac{1}{\varepsilon} \partial_{3} u_{n}=0, \\
\left(\left.u_{n}\right|_{t=0},\left.b_{n}\right|_{t=0}\right)=\left(J_{n} u_{0}, J_{n} b_{0}\right) .
\end{gathered}
$$

The following product law will be useful throughout this paper (see [3]).

Lemma 2.1. Let $\sigma>3 / 2+2$ be an integer. There exists a constant $C$ such that for all vector fields $a$ and $b$ in $H^{\sigma}(\Omega)$ satisfying $\operatorname{div} a=0$,

$$
\begin{aligned}
& \left|\prec a \cdot \nabla b, b \succ_{H^{\sigma}(\Omega)}\right| \\
& \quad \leq C\|\nabla b\|_{H^{\sigma-1}(\Omega)}\left(\|\nabla a\|_{H^{\sigma-1}(\Omega)}\|\nabla b\|_{H^{\sigma-2}(\Omega)}+\|\nabla a\|_{H^{\sigma-2}(\Omega)}\|\nabla b\|_{H^{\sigma-1}(\Omega)}\right) .
\end{aligned}
$$

Moreover, if $\operatorname{div} b=0$, then

$$
\begin{aligned}
& \left|\prec b \cdot \nabla a, b \succ_{H^{\sigma}(\Omega)}+\prec b \cdot \nabla b, a \succ_{H^{\sigma}(\Omega)}\right| \\
& \quad \leq C\|\nabla b\|_{H^{\sigma-1}(\Omega)}\left(\|\nabla a\|_{H^{\sigma-1}(\Omega)}\|\nabla b\|_{H^{\sigma-2}(\Omega)}+\|\nabla a\|_{H^{\sigma-2}(\Omega)}\|\nabla b\|_{H^{\sigma-1}(\Omega)}\right) .
\end{aligned}
$$

Here the symbol $\prec \cdot, \cdot \succ_{H^{\sigma}(\Omega)}$ denotes the scalar product in $H^{\sigma}(\Omega)$.

To continue the proof of Theorem 1.1, we take the scalar product in $H^{s}(\Omega)$ and we use the above lemma to obtain, for all $t \in\left[0, T_{n}^{*}(\varepsilon)[\right.$,

$$
\begin{aligned}
& \left\|U_{n}(t)\right\|_{H^{s}(\Omega)}^{2}+2 \varepsilon \int_{0}^{t}\left\|\nabla u_{n}(\tau)\right\|_{H^{s}(\Omega)}^{2} d \tau+\frac{2}{\varepsilon} \int_{0}^{t}\left\|\nabla b_{n}(\tau)\right\|_{H^{s}(\Omega)}^{2} d \tau \\
& \leq\left\|U_{0}\right\|_{H^{s}(\Omega)}^{2}+C \int_{0}^{t}\left\|\nabla U_{n}(\tau)\right\|_{H^{s-2}(\Omega)}\left\|\nabla U_{n}(\tau)\right\|_{H^{s-1}(\Omega)}^{2} d \tau .
\end{aligned}
$$

Set

$$
T(n, \varepsilon):=\sup \left\{0 \leq t<T_{n}^{*}(\varepsilon) ; \forall \tau \in[0, t]\left\|U_{n}(\tau)\right\|_{H^{s}(\Omega)} \leq 2\left\|U_{0}\right\|_{H^{s}(\Omega)}\right\} .
$$

Using (2.7) and Gronwall's lemma, we obtain, for all $t \in[0, T(n, \varepsilon)[$,

$$
\left\|U_{n}(t)\right\|_{H^{s}(\Omega)}^{2} \leq\left\|U_{0}\right\|_{H^{s}(\Omega)}^{2} \exp \left(2 C t\left\|U_{0}\right\|_{H^{s}(\Omega)}\right) .
$$


Thus

$$
T(n, \varepsilon)>T:=\frac{\log (\sqrt{3})}{2 C\left\|U_{0}\right\|_{H^{s}(\Omega)}}>0 .
$$

Moreover, for all $t \in[0, T]$,

$$
\left\|U_{n}(t)\right\|_{H^{s}(\Omega)}^{2}+2 \varepsilon \int_{0}^{t}\left\|\nabla u_{n}(\tau)\right\|_{H^{s}(\Omega)}^{2} d \tau+\frac{2}{\varepsilon} \int_{0}^{t}\left\|\nabla b_{n}(\tau)\right\|_{H^{s}(\Omega)}^{2} d \tau \leq 5\left\|U_{0}\right\|_{H^{s}(\Omega)}^{2} .
$$

Using Ascoli's theorem, the Cantor diagonal process, and the estimate (2.11), for $n$ tends to infinity, we obtain a solution that satisfies the following estimate:

$$
\begin{aligned}
& \left\|U^{\varepsilon}(t)\right\|_{H^{s}(\Omega)}^{2}+2 \varepsilon \int_{0}^{t}\left\|\nabla u^{\varepsilon}(\tau)\right\|_{H^{s}(\Omega)}^{2} d \tau+\frac{2}{\varepsilon} \int_{0}^{t}\left\|\nabla b^{\varepsilon}(\tau)\right\|_{H^{s}(\Omega)}^{2} d \tau \\
& \leq 5\left\|U_{0}\right\|_{H^{s}(\Omega)}^{2}, \quad \forall t \in[0, T] .
\end{aligned}
$$

This regularity implies in a standard way the uniqueness.

It remains to prove the global existence when the initial data is small enough. We now assume that $\left\|U_{0}\right\|_{H^{s}(\Omega)} \leq c \mathcal{E},(c=1 / C)$, and we set

$$
T_{n}(\varepsilon):=\sup \left\{0 \leq t<T_{n}^{*}(\varepsilon) ; \forall \tau \in[0, t],\left\|U_{n}(\tau)\right\|_{H^{s}(\Omega)} \leq c \varepsilon\right\} .
$$

By (2.11), we have $d / d t\left\|U_{n}\right\|_{H^{s}(\Omega)}^{2}(0)<0$, then there exists $t_{n}>0$ such that $\left\|U_{n}\left(t_{n}\right)\right\|_{H^{s}(\Omega)}$ $<c \varepsilon$. Finally, since $\left\|U_{n}(t)\right\|_{H^{s}(\Omega)}$ is a decreasing time function on $\left[t_{n}, T_{n}^{*}(\varepsilon)\right.$ [, one concludes that $T_{n}(\varepsilon)=T_{n}^{*}(\varepsilon)$.

\section{Study of the periodic case}

In this section, we will prove Theorem 1.2 stated in the introduction. The assertion (1.11) is obvious and follows directly from the energy estimate. For the equality (1.12), it suffices to integrate the second MHD equation in the space variable $x$ over the torus $\mathbb{T}^{3}$. To prove (1.13), we start by writing $\left\|u_{\mathrm{osc}}\right\|_{H^{\sigma}\left(\mathbb{T}^{3}\right)}$ in terms of the Fourier expansion

$$
\left\|u_{\mathrm{osc}}\right\|_{H^{\sigma}\left(\mathbb{T}^{3}\right)}^{2}=\sum_{\substack{k=\left(k_{1}, k_{2}, k_{3}\right) \\ k_{2} \neq 0}}\left(1+|k|^{2}\right)^{\sigma}|\hat{u}(k)|^{2} .
$$

Then we have just to estimate $\left|\widehat{u}^{\varepsilon}(t, k)\right|$ for $k_{2} \neq 0$. To do so, we observe that for $k_{2} \neq 0$, $\hat{\mathcal{u}}^{\varepsilon}(\cdot, k)$ is a solution of an ordinary differential equation. First of all, we rewrite the system $\left(\mathscr{S}^{\mathcal{E}}\right)$ in the following form:

$$
\partial_{t} U^{\varepsilon}+\frac{1}{\varepsilon} M\left(U^{\varepsilon}\right)=F^{\varepsilon}
$$

where

$$
\begin{gathered}
\mathcal{M}\left(U^{\varepsilon}\right):=\left(-\partial_{2} b^{\varepsilon}+\mathbb{P}\left(u^{\varepsilon} \times e_{3}\right),-\partial_{2} u^{\varepsilon}-\Delta b^{\varepsilon}\right) \\
F^{\varepsilon}=\left(\varepsilon \Delta u^{\varepsilon}-\mathbb{P}\left(u^{\varepsilon} \cdot \nabla u^{\varepsilon}\right)+\mathbb{P}\left(b^{\varepsilon} \cdot \nabla b^{\varepsilon}\right), b^{\varepsilon} \cdot \nabla u^{\varepsilon}-u^{\varepsilon} \cdot \nabla b^{\varepsilon}\right)=\left(F_{1}^{\varepsilon}, F_{2}^{\varepsilon}\right) .
\end{gathered}
$$


The energy estimate implies that the family $\left(F^{\varepsilon}\right)$ is bounded in $L^{2}\left([0, T], H^{s-1}\left(\mathbb{T}^{3}\right)\right)$. If we apply the operator "curl" to the first equation, by the partial Fourier transform with respect to the space variable, we get

$$
\begin{gathered}
\partial_{t}\left(i M(k) \widehat{u^{\varepsilon}}(t, k)\right)+i \frac{k_{3}}{\varepsilon} \widehat{u^{\varepsilon}}(t, k)-\frac{k_{2}}{\varepsilon} M(k) \widehat{b^{\varepsilon}}(t, k)=i M(k) \widehat{F_{1}^{\varepsilon}}(t, k), \\
\partial_{t} \widehat{b^{\varepsilon}}(t, k)+i \frac{k_{2}}{\varepsilon} \widehat{u}^{\varepsilon}(t, k)+\frac{|k|^{2}}{\varepsilon} \widehat{b^{\varepsilon}}(t, k)=\widehat{F_{2}^{\varepsilon}}(t, k),
\end{gathered}
$$

where

$$
M(k)=\left(\begin{array}{ccc}
0 & -k_{3} & k_{2} \\
k_{3} & 0 & -k_{1} \\
-k_{2} & k_{1} & 0
\end{array}\right) .
$$

We recall that the eigenvalues of $M(k)$ are $\pm i|k|, 0$ and the corresponding eigenvectors are $\nu(k)^{ \pm}$and $k /|k|$ which is not divergence-free. In this eigenbase, the velocity field $u^{\varepsilon}$ and the magnetic perturbation $b^{\varepsilon}$ can be written as follow:

$$
\begin{aligned}
& \hat{u}^{\varepsilon}(t, k)={\widehat{u^{\varepsilon}}}^{+}(t, k) v(k)^{+}+{\widehat{u^{\varepsilon}}}^{-}(t, k) v(k)^{-}, \\
& \hat{b}^{\varepsilon}(t, k)=\hat{b}^{+}(t, k) v(k)^{+}+\widehat{b}^{-}(t, k) v(k)^{-} .
\end{aligned}
$$

In terms of variables $\widehat{\mathcal{u}}^{\varepsilon},{\widehat{\mathcal{u}^{\varepsilon}}}^{-}, \hat{b}^{\varepsilon}$, and ${\hat{b^{\varepsilon}}}^{-},(3.4)$ splits into four equations which can be summarized in

$$
\partial_{t}{\widehat{U^{\varepsilon}}}^{ \pm}(t, k)+\frac{1}{\varepsilon} A^{ \pm}(k) \widehat{U}^{ \pm}(t, k)=\widehat{F}^{ \pm}(t, k)
$$

where

$$
A^{ \pm}(k)=\left(\begin{array}{cc}
\mp i \frac{k_{3}}{|k|} & i k_{2} \\
i k_{2} & |k|^{2}
\end{array}\right)
$$

With a direct computation, one can prove the following lemma.

Lemma 3.1. Let $\lambda_{j}^{ \pm}(k), j=1,2$, be the eigenvalues of $A^{ \pm}(k)$. Then

$$
\begin{aligned}
& \lambda_{1}^{ \pm}(k)=\frac{1}{2}\left[\left(|k|^{2}+\alpha(k)\right) \pm i\left(\beta(k)-\frac{k_{3}}{|k|}\right)\right], \\
& \lambda_{2}^{ \pm}(k)=\frac{1}{2}\left[\left(|k|^{2}-\alpha(k)\right) \pm i\left(\beta(k)+\frac{k_{3}}{|k|}\right)\right],
\end{aligned}
$$

where

$$
(\alpha(k)+i \beta(k))^{2}=|k|^{4}-\frac{k_{3}^{2}}{|k|^{2}}-4 k_{2}^{2}+2 i k_{3}|k|, \quad \alpha(k) \geq 0 .
$$


Moreover, if we denote by $\omega_{j}^{ \pm}(k)$ the real part of $\lambda_{j}^{ \pm}(k)$, it holds that

$$
\omega_{j}^{ \pm}(k)>0 \Longleftrightarrow k_{2} \neq 0
$$

By Duhamel's formula, we have

$$
{\widehat{U^{\varepsilon}}}^{ \pm}(t, k)=\exp \left(-\frac{t}{\varepsilon} A^{ \pm}(k)\right){\widehat{U_{0}}}^{ \pm}(k)+\int_{0}^{t} \exp \left(-\frac{t-\tau}{\varepsilon} A^{ \pm}(k)\right) \widehat{F}^{ \pm}{ }^{ \pm}(\tau, k) d \tau .
$$

Let $\omega(k):=\operatorname{Min}\left(\omega_{j}^{ \pm}(k)\right)$. Using the Cauchy-Schwarz inequality, we obtain, for $k_{2} \neq 0$,

$$
\left|\widehat{U^{\varepsilon}}(t, k)\right|^{2} \leq 2 \exp \left(-\frac{2 t}{\varepsilon} \omega(k)\right)\left|\widehat{U_{0}}(k)\right|^{2}+\frac{\varepsilon}{\omega(k)}\left(1-\exp \left(-\frac{T}{\varepsilon} \omega(k)\right)\right) \int_{0}^{t}\left|\widehat{F^{\varepsilon}}(\tau, k)\right|^{2} d \tau \text {. }
$$

For the case of low frequencies, since $\left(F^{\varepsilon}\right)$ is bounded in $L^{2}\left([0, T], H^{s-1}\right)$, then for all $\delta \in] 0, T]$ we have

$$
\forall k \in \mathbb{Z}^{3} ; k_{2} \neq 0 \quad \sup _{\delta \leq t \leq T}\left|\widehat{U^{\varepsilon}}(t, k)\right|^{2} \longrightarrow 0 \text { as } \varepsilon \longrightarrow 0
$$

Now, we have to study the high frequencies. Let $n \in \mathbb{N}^{*}$ and $k \in \mathbb{Z}^{3}$ with $|k| \geq n$. For $t \in[0, T]$, the MHD system gives

$$
\begin{aligned}
& \left|\hat{u}^{\varepsilon}(t, k)\right|^{2}-\left|\widehat{u_{0}}(k)\right|^{2}+\left|\hat{b}^{\varepsilon}(t, k)\right|^{2}-\left|\widehat{b_{0}}(k)\right|^{2}+\varepsilon \int_{0}^{t}|k|^{2}\left|\widehat{u}^{\varepsilon}(\tau, k)\right|^{2} d \tau \\
& \quad+\frac{2}{\varepsilon} \int_{0}^{t}|k|^{2}\left|\widehat{b}^{\varepsilon}(\tau, k)\right|^{2} d \tau+\frac{2}{\varepsilon} \int_{0}^{t}\left(\widehat{\partial_{3} b^{\varepsilon}}(\tau, k) \overline{\hat{u}^{\varepsilon}}(\tau, k)+\widehat{\partial_{3} u^{\varepsilon}}(\tau, k) \overline{\hat{b}^{\varepsilon}}(\tau, k)\right) d \tau \\
& =\int_{0}^{t} \widehat{b^{\varepsilon} \cdot \nabla b^{\varepsilon}}(\tau, k) \overline{\hat{u}^{\varepsilon}}(\tau, k)+\widehat{u^{\varepsilon} \cdot \nabla b^{\varepsilon}}(\tau, k) \overline{\hat{b}^{\varepsilon}}(\tau, k)+\widehat{b^{\varepsilon} \cdot \nabla u^{\varepsilon}}(\tau, k) \overline{\hat{b}^{\varepsilon}}(\tau, k) d \tau \\
& \quad-\int_{0}^{t} \widehat{u^{\varepsilon} \cdot \nabla u^{\varepsilon}}(\tau, k) \overline{\hat{u}^{\varepsilon}}(\tau, k) d \tau .
\end{aligned}
$$

First, the energy estimate and the condition $s>3 / 2+1$ imply that

$$
\left|\sum_{|k| \geq n} \int_{0}^{t}\left(\widehat{b^{\varepsilon \cdot \nabla b}} \varepsilon(\tau, k) \overline{\hat{u}^{\varepsilon}}(\tau, k)+\widehat{u^{\varepsilon} \cdot \nabla b} \varepsilon(\tau, k) \overline{\hat{b}^{\varepsilon}}(\tau, k)+\widehat{b^{\varepsilon} \cdot \nabla u^{\varepsilon}}(\tau, k) \overline{\hat{b}^{\varepsilon}}(\tau, k)\right) d \tau\right| \leq C \varepsilon^{1 / 2} .
$$

On the other hand, we remark that

$$
\sum_{|k| \geq n} \int_{0}^{t}\left(\widehat{\partial_{3} b^{\varepsilon}}(\tau, k) \overline{\hat{u}^{\varepsilon}}(\tau, k)+\widehat{\partial_{3} u^{\varepsilon}}(\tau, k) \overline{\hat{b}^{\varepsilon}}(\tau, k)\right) d \tau=0 .
$$


It remains to study the following cubic term:

$$
\sum_{|k| \geq n} \int_{0}^{t} \widehat{u^{\varepsilon} \cdot \nabla u^{\varepsilon}}(\tau, k) \overline{\hat{u}^{\varepsilon}}(\tau, k) d \tau .
$$

As in [2], we begin by mentioning that for $|k| \geq n$ we have

$$
\left|\widehat{u^{\varepsilon} \cdot \nabla u^{\varepsilon}}(\tau, k)\right| \leq \sum_{|p| \leq|k| / 2}\left|\widehat{u}^{\varepsilon}(\tau, p)\right|\left|\widehat{\nabla u^{\varepsilon}}(\tau, k-p)\right|+\sum_{|p| \geq|k| / 2}\left|\widehat{u^{\varepsilon}}(\tau, p)\right|\left|\widehat{\nabla u^{\varepsilon}}(\tau, k-p)\right| .
$$

The Cauchy-Schwarz inequality yields

$$
\begin{aligned}
\left|\widehat{u^{\varepsilon} \cdot \nabla u^{\varepsilon}}(\tau, k)\right| \leq & \|\left. u^{\varepsilon}(\tau)\right|_{L^{2}\left(\mathbb{T}^{3}\right)}\left(\sum_{|p| \leq|k| / 2}\left|\widehat{\nabla u^{\varepsilon}}(\tau, k-p)\right|^{2}\right)^{1 / 2} \\
& +\left\|\nabla u^{\varepsilon}(\tau)\right\|_{L^{2}\left(\mathbb{T}^{3}\right)}\left(\sum_{|p| \geq|k| / 2}\left|\widehat{u^{\varepsilon}}(\tau, p)\right|^{2}\right)^{1 / 2} \\
\leq & 2\left\|u^{\varepsilon}(\tau)\right\|_{H^{1}\left(\mathbb{T}^{3}\right)}|| u_{k}^{\varepsilon}(\tau) \|_{H^{1}\left(\mathbb{T}^{3}\right)^{\prime}}
\end{aligned}
$$

where

$$
\left\|u_{k}^{\varepsilon}(\tau)\right\|_{H^{1}\left(\mathbb{T}^{3}\right)}^{2}:=\sum_{|p| \geq|k| / 2}|p|^{2}\left|\widehat{u}^{\varepsilon}(\tau, p)\right|^{2}
$$

Using the energy estimate (1.8), we obtain

$$
\begin{aligned}
\left|\sum_{|k| \geq n} \widehat{u^{\varepsilon} \cdot \nabla u^{\varepsilon}}(\tau, k) \overline{\hat{u}^{\varepsilon}}(\tau, k)\right| & \leq C \sum_{|k| \geq n} \|\left. u_{k}^{\varepsilon}(\tau)\right|_{H^{1}\left(\mathbb{T}^{3}\right)}\left|{\widehat{u^{\varepsilon}}}(\tau, k)\right| \\
& \leq C\left(\sum_{|k| \geq n}\left\|u_{k}^{\varepsilon}(\tau)\right\|_{H^{1}\left(\mathbb{T}^{3}\right)}^{2}+\sum_{|k| \geq n}\left|\hat{u}^{\varepsilon}(\tau, k)\right|^{2}\right) .
\end{aligned}
$$

Note that, for $\alpha \in] 3 / 2, s-1$ ], we have

$$
\begin{aligned}
\sum_{|k| \geq n}\left\|u_{k}^{\varepsilon}(\tau)\right\|_{H^{1}\left(\mathbb{T}^{3}\right)}^{2} & =\sum_{|k| \geq n} \sum_{|p| \geq|k| / 2}|p|^{2}\left|{\widehat{u^{\varepsilon}}}^{\varepsilon}(\tau, p)\right|^{2} \\
& \leq 2^{2 \alpha} \sum_{|k| \geq n} \frac{1}{|k|^{2 \alpha}} \sum_{|p| \geq|k| / 2}|p|^{2(\alpha+1)}\left|\hat{\mathcal{u}}^{\varepsilon}(\tau, p)\right|^{2} \leq C \sum_{|k| \geq n} \frac{1}{|k|^{2 \alpha}} .
\end{aligned}
$$

If we denote $\eta_{n}:=C \sum_{|k| \geq n} 1 /|k|^{2 \alpha}$, then $\eta_{n} \underset{n \rightarrow+\infty}{\longrightarrow} 0$. 
The above inequality, together with (3.16), (3.22), and (3.23), gives

$$
\sum_{|k| \geq n}\left|\hat{u}^{\varepsilon}(t, k)\right|^{2} \leq C \varepsilon^{1 / 2}+T \eta_{n}+r_{n}+\int_{0}^{t} \sum_{|k| \geq n}\left|\widehat{u}^{\varepsilon}(\tau, k)\right|^{2} d \tau,
$$

where $r_{n}=\sum|k| \geq n\left(\left|\widehat{u_{0}}(k)\right|^{2}+\left|\widehat{b_{0}}(k)\right|^{2}\right)$.

The Gronwall lemma implies that, for all $t \in[0, T]$,

$$
\sum_{|k| \geq n}\left|\hat{u}^{\varepsilon}(t, k)\right|^{2} \leq\left(C \varepsilon^{1 / 2}+T \eta_{n}+r_{n}\right) \exp (T)
$$

It follows that

$$
\underset{\varepsilon \rightarrow 0}{\limsup }\left\|u_{\mathrm{osc}}^{\varepsilon}\right\|_{L^{\infty}\left([0, T], L^{2}\left(\mathbb{T}^{3}\right)\right)} \leq\left(T \eta_{n}+r_{n}\right) \exp (T) .
$$

By interpolation argument, we complete the proof of (1.13).

Now we turn to study the average case $\left(k_{2}=0\right)$. We integrate the first equation of MHD system with respect to the second space variable $x_{2}$. We obtain an average equation, in which the term $\mathbb{P}\left(\operatorname{curl}(b) \times e_{2}\right)$ disappears and the penalization operator becomes $L(v)=$ $\mathbb{P}\left(v \times e_{3}\right)$. If we denote $g^{\varepsilon}=\varepsilon \Delta \overline{\mathcal{u}^{\varepsilon}}+\overline{\mathbb{P}\left(b^{\varepsilon} \cdot \nabla b^{\varepsilon}\right)}$, we obtain

$$
\begin{gathered}
\partial_{t} \overline{u^{\varepsilon}}+\overline{\mathbb{P}\left(u^{\varepsilon} \cdot \nabla u^{\varepsilon}\right)}+\frac{1}{\varepsilon} \mathbb{P}\left(\overline{u^{\varepsilon}} \times e_{3}\right)=g^{\varepsilon} \quad \text { in }[0, T] \times \mathbb{T}^{2} \\
\partial_{1} \overline{u_{1}^{\varepsilon}}+\partial_{3} \overline{u_{3}^{\varepsilon}}=0,\left.\quad \overline{u^{\varepsilon}}\right|_{t=0}=\overline{u_{0}} .
\end{gathered}
$$

The energy estimate implies that

$$
g^{\varepsilon} \longrightarrow 0 \quad \text { in } L^{2}\left([0, T], H^{s-1}\left(\mathbb{T}^{2}\right)\right)
$$

Using the decomposition $u^{\varepsilon}=\overline{u^{\varepsilon}}+u_{\text {osc }}^{\varepsilon}$, by a direct Fourier computation, one proves that the attraction between "slow" waves $\overline{\mathcal{u}^{\varepsilon}}$ and "fast" ones $u_{\mathrm{osc}}^{\varepsilon}$ has no resultant; that is $\overline{u^{\varepsilon}}$. $\nabla u_{\mathrm{osc}}^{\varepsilon}=u_{\mathrm{osc}}^{\varepsilon} \cdot \nabla \overline{u^{\varepsilon}}=0$. However, "fast" waves interfere to create additional ones given by $u_{\mathrm{osc}}^{\varepsilon} \cdot \nabla u_{\mathrm{osc}}^{\varepsilon}$.

Denote $G^{\varepsilon}=g^{\varepsilon}-\overline{\mathbb{P}\left(u_{\mathrm{osc}}^{\varepsilon} \cdot \nabla u_{\mathrm{osc}}^{\varepsilon}\right)}$. System (3.27) becomes

$$
\begin{gathered}
\partial_{t} \overline{u^{\varepsilon}}+\mathbb{P}\left(\overline{u^{\varepsilon}} \cdot \nabla \overline{u^{\varepsilon}}\right)+\frac{1}{\varepsilon} \mathbb{P}\left(\overline{u^{\varepsilon}} \times e_{3}\right)=G^{\varepsilon} \quad \text { in }[0, T] \times \mathbb{T}^{2}, \\
\partial_{1} \overline{u_{1}^{\varepsilon}}+\partial_{3} \overline{u_{3}^{\varepsilon}}=0,\left.\quad \overline{u^{\varepsilon}}\right|_{t=0}=\overline{u_{0}} .
\end{gathered}
$$

The idea is to filter the average system (3.29) by the group $\mathscr{L}(t)$ associated to $L=\mathbb{P}(u \times$ $\left.e_{3}\right)$. Although the operator $L$ is well known in the literature, we recall in the following lemma some of its properties which are useful in our purpose.

Lemma 3.2. The wave equation

$$
\begin{gathered}
\partial_{t} u+L(u)=0 \quad \text { in } \mathbb{R} \times \mathbb{T}^{2}, \\
\partial_{1} u_{1}+\partial_{3} u_{3}=0, \quad u(0)=u_{0}
\end{gathered}
$$


has a global solution denoted by $u(t)=\mathscr{L}(t) u_{0}$, satisfying for all $s \in \mathbb{R}$ and for all $u_{0}$ in $H^{s}\left(\mathbb{T}^{2}\right)$,

$$
\begin{aligned}
\| \mathscr{L}(t) u_{0} & \left\|_{H^{s}\left(\mathbb{T}^{2}\right)}=\right\| u_{0} \|_{H^{s}\left(\mathbb{T}^{2}\right)}, \\
\|^{t} \mathscr{L}(t) u_{0} & \left\|_{H^{s}\left(\mathbb{T}^{2}\right)}=\right\| u_{0} \|_{H^{s}\left(\mathbb{T}^{2}\right)} .
\end{aligned}
$$

Proof. A classical computation leads to

$$
\widehat{u}(t, k)=\exp (i \omega(k) t)\left(\widehat{u_{0}}(k) / \nu(k)^{+}\right) \nu(k)^{+}+\exp (-i \omega(k) t)\left(\widehat{u_{0}}(k) / v(k)^{-}\right) \nu(k)^{-} \text {, }
$$

where

$$
\begin{aligned}
& \omega(k)=\frac{k_{3}}{\left(k_{1}^{2}+k_{3}^{2}\right)^{1 / 2}}, \\
& \nu(k)^{+}= \begin{cases}\left(1,-i \frac{k_{3}}{\left|k_{3}\right|}, 0\right) & \text { if } k_{1}=0, \\
\frac{(\alpha(k), \beta(k), \gamma(k))}{\left(|\alpha(k)|^{2}+|\beta(k)|^{2}+|\gamma(k)|^{2}\right)^{1 / 2}} & \text { if } k_{1} \neq 0,\end{cases} \\
& (\alpha(k), \beta(k), \gamma(k))=\left(-k_{1} k_{3}, i k_{1}\left(k_{1}^{2}+k_{3}^{2}\right)^{1 / 2}, k_{1}^{2}\right), \\
& v(k)^{-}=\overline{v(k)^{+}} .
\end{aligned}
$$

Denote $v^{\varepsilon}(t):=\mathscr{L}(-t / \varepsilon) \overline{u^{\varepsilon}}(t)$. System (3.29) becomes

$$
\begin{gathered}
\partial_{t} v^{\varepsilon}+\mathscr{L}\left(-\frac{t}{\varepsilon}\right) \mathbb{P}\left(\mathscr{L}\left(\frac{t}{\varepsilon}\right) v^{\varepsilon} \cdot \nabla \mathscr{L}\left(\frac{t}{\varepsilon}\right) v^{\varepsilon}\right)=K^{\varepsilon} \quad \text { in }[0, T] \times \mathbb{T}^{2}, \\
\partial_{1} v_{1}^{\varepsilon}+\partial_{3} v_{3}^{\varepsilon}=0,\left.\quad v^{\varepsilon}\right|_{t=0}=\overline{u_{0}},
\end{gathered}
$$

where $K^{\varepsilon}=\mathscr{L}(-t / \varepsilon) G^{\varepsilon}$.

System (3.34) can be rewritten as follows:

$$
\begin{gathered}
\partial_{t} v^{\varepsilon}+Q^{\varepsilon}\left(v^{\varepsilon}, v^{\varepsilon}\right)=K^{\varepsilon} \text { in }[0, T] \times \mathbb{T}^{2}, \\
\partial_{1} v_{1}^{\varepsilon}+\partial_{3} v_{3}^{\varepsilon}=0, \\
\left.v^{\varepsilon}\right|_{t=0}=\overline{u_{0}},
\end{gathered}
$$

where the filtered quadratic form is given by

$$
Q^{\varepsilon}(v, v)=\mathscr{L}\left(-\frac{t}{\varepsilon}\right) \mathbb{P}\left(\mathscr{L}\left(\frac{t}{\varepsilon}\right) v \cdot \nabla \mathscr{L}\left(\frac{t}{\varepsilon}\right) v\right) .
$$


Let $Q^{0}=\lim _{\varepsilon \rightarrow 0} Q^{\varepsilon}$ in the sense of distributions. We obtain formally the following Eulertype limit system:

$$
\begin{gathered}
\partial_{t} v+Q^{0}(v, v)=0 \quad \text { in }[0, T] \times \mathbb{T}^{2}, \\
\partial_{1} v_{1}+\partial_{3} v_{3}=0, \\
\left.v\right|_{t=0}=\overline{u_{0}},
\end{gathered}
$$

for which we establish the following theorem.

Theorem 3.3. Let $s>2 / 2+2$ be an integer and $U_{0}=\left(u_{0}, b_{0}\right) \in H^{s}\left(\mathbb{T}^{3}\right)$ a pair of divergencefree fields. Then, there exist $T>0$ and a unique solution $v$ of $(\mathscr{L} \mathscr{S})$ in the space $\mathscr{C}^{0}([0, T]$, $\left.H^{s}\left(\mathbb{T}^{2}\right)\right)$ with initial data $\overline{u_{0}}$ satisfying

$$
\|v\|_{H^{s}\left(\mathbb{T}^{2}\right)}^{2} \leq\left\|\overline{\mathcal{u}_{0}}\right\|_{H^{s}\left(\mathbb{T}^{2}\right)}^{2} .
$$

Proof. We have to estimate the term $\left|\int_{0}^{t} \prec Q^{0}\left(v_{n}, v_{n}\right), v_{n} \succ_{H^{s}\left(\mathbb{T}^{2}\right)}\right|$, where $v_{n}$ is the solution of the approximate limit system. Since $\lim _{\mathcal{\varepsilon} \rightarrow 0} \int_{0}^{t} \prec Q^{\varepsilon}\left(v_{n}, v_{n}\right), v_{n} \succ_{H^{s}\left(\mathbb{T}^{2}\right)}=\int_{0}^{t} \prec Q^{0}\left(v_{n}, v_{n}\right)$, $v_{n} \succ_{H^{s}\left(\mathbb{T}^{2}\right)}$, it holds as in the proof of the existence result that

$$
\left|\int_{0}^{t} \prec Q^{0}\left(v_{n}, v_{n}\right), v_{n} \succ_{H^{s}\left(\mathbb{T}^{2}\right)}\right| \leq C \int_{0}^{t}\left\|\nabla v_{n}(\tau)\right\|_{H^{s-2}\left(\mathbb{T}^{2}\right)}\left\|\nabla v_{n}(\tau)\right\|_{H^{s-1}\left(\mathbb{T}^{2}\right)}^{2} d \tau \text {. }
$$

Remark 3.4. There are many papers dealing with the possible relation between the life span of the solution of the considered system (such as $\left(\mathscr{S}^{\varepsilon}\right)$ ) and the life span of the solution of the corresponding limit system (such as $(\mathscr{L} \mathscr{S})$ ), especially how to recuperate global well-posedness or uniform local well-posedness of the considered system from the limit one, at least for $\varepsilon$ small enough (see, e.g., $[5,9,10,17])$. However, since we will focus on justifying the convergence result, any uniform time of existence of both $\left(\mathscr{S}^{\varepsilon}\right)$ and $(\mathscr{L} \mathscr{S})$, denoted also by $T$, do the job.

Let us now turn to the proof of the convergence result. Denote $W^{\varepsilon}=v^{\varepsilon}-v$. We obtain

$$
\begin{gathered}
\partial_{t} W^{\varepsilon}+Q^{\varepsilon}\left(W^{\varepsilon}, W^{\varepsilon}+2 v\right)=K^{\varepsilon}-R_{\mathrm{osc}}^{\varepsilon} \quad \text { in }[0, T] \times \mathbb{T}^{2}, \\
\partial_{1} W_{1}^{\varepsilon}+\partial_{3} W_{3}^{\varepsilon}=0,\left.\quad W^{\varepsilon}\right|_{t=0}=0,
\end{gathered}
$$

where

$$
R_{\mathrm{osc}}^{\varepsilon}=Q^{\varepsilon}(v, v)-Q^{0}(v, v) .
$$

By the nonstationary phases theorem, we get

$$
\widehat{R_{\mathrm{osc}}^{\varepsilon}}(t, n)=\sum_{\sigma \in\{ \pm\}^{3}} \sum_{\substack{k+m=n \\ n_{3} \neq 0 \\ \omega_{n, k, m} \neq 0}} e^{-i(t / \varepsilon) \omega_{n, k, m}}\left(\widehat{v}^{\sigma_{1}}(t, k) \cdot \widehat{\nabla v}^{\sigma_{2}}(t, m)\right)^{\sigma_{3}}(n),
$$


where

$$
\begin{gathered}
\sigma=\left(\sigma_{1}, \sigma_{2}, \sigma_{3}\right), \\
\omega_{n, k, m}=\sigma_{1} \frac{n_{3}}{|n|}-\sigma_{2} \frac{k_{3}}{|k|}-\sigma_{3} \frac{m_{3}}{|m|},
\end{gathered}
$$

and, for any vector $X$,

$$
X^{ \pm}(n)=\left(X / \nu^{ \pm}(n)\right) \nu^{ \pm}(n)
$$

The right-hand side of the above system will be composed of two terms. The first is $K^{\varepsilon}$, which tends to zero in $L^{2}\left([0, T], H^{s-2}\left(\mathbb{T}^{2}\right)\right)$ when $\varepsilon \rightarrow 0$. The second is the oscillating term $R_{\mathrm{osc}}^{\varepsilon}$, which converges weakly to zero but not strongly. The method we use here to deal with $R_{\mathrm{osc}}^{\varepsilon}$ is inspired from the ideas introduced by Schochet in [19] and applied by Gallagher in $[9,10]$. The idea consists in dividing $R_{\mathrm{osc}}^{\varepsilon}$ into high-frequency term $R_{\mathrm{osc}}^{\varepsilon, N}$ and low-frequency term $R_{\mathrm{osc}, N}^{\varepsilon}$ defined, respectively, for any arbitrary cutoff integer $N \geq 1$, by

$$
\begin{aligned}
& R_{\mathrm{osc}}^{\varepsilon, N}=R_{\mathrm{osc}}^{\varepsilon}-R_{\mathrm{osc}, N}^{\varepsilon}, \\
& \widehat{R_{\mathrm{osc}, N}}(t, n)=\mathbf{1}_{\{|n| \leq N\}} \sum_{\sigma \in\{ \pm\}^{3}} \sum_{\substack{k+m=n, n_{3} \neq 0 \\
\omega_{n, k, m} \neq 0 \\
|k|,|m| \leq N}} e^{-i(t / \varepsilon) \omega_{n, k, m}}\left(\widehat{v}^{\sigma_{1}}(t, k) \cdot \widehat{\nabla v}^{\sigma_{2}}(t, m)\right)^{\sigma_{3}}(n) .
\end{aligned}
$$

In a first time, we consider the case of high frequencies. We recall the following lemma (see [10]).

Lemma 3.5. Let $s \in \mathbb{R}$. For any $a \in \mathscr{C}^{0}\left([0, T], H^{s}\left(\mathbb{T}^{2}\right)\right)$, the high-frequency part $a^{N}$, defined by $\widehat{a^{N}}=\mathbf{1}_{[N,+\infty}\left[\hat{a}\right.$, tends to zero in $\mathscr{C}^{0}\left([0, T], H^{s}\left(\mathbb{T}^{2}\right)\right)$ when $N$ tends to infinity.

This lemma leads to the following proposition.

Proposition 3.6. The high-frequency term $R_{\text {osc }}^{\varepsilon, N}$ tends to zero in $\mathscr{C}^{0}\left([0, T], H^{s-2}\left(\mathbb{T}^{2}\right)\right)$, uniformly in $\varepsilon$, when $N$ tends to infinity.

To absorb the low-frequency term, we adopt the following change of function:

$$
\varphi_{N}^{\varepsilon}=W^{\varepsilon}+\varepsilon \widetilde{R}_{\mathrm{osc}, N}^{\varepsilon}
$$

where

$$
\widehat{\widetilde{R}_{\mathrm{osc}, N}^{\varepsilon}}=\mathbf{1}_{\{|n| \leq N\}} \sum_{\sigma \in\{ \pm\}^{3}} \sum_{\substack{k+m=n, n_{3} \neq 0 \\ \omega_{n, k, m} \neq 0 \\|k|,|m| \leq N}} \frac{e^{-i(t / \varepsilon) \omega_{n, k, m}}}{\omega_{n, k, m}}\left(\hat{v}^{\sigma_{1}}(t, k) \cdot \widehat{\nabla v}^{\sigma_{2}}(t, m)\right)^{\sigma_{3}}(n) .
$$

It is easy to see that $\varphi_{N}^{\varepsilon}$ satisfies the following equation:

$$
\partial_{t} \varphi_{N}^{\varepsilon}+Q^{\varepsilon}\left(\varphi_{N}^{\varepsilon}, \varphi_{N}^{\varepsilon}-2 \varepsilon \widetilde{R}_{\mathrm{osc}, N}^{\varepsilon}+2 v\right)=K^{\varepsilon}-R_{\mathrm{osc}}^{\varepsilon, N}+\varepsilon r_{\mathrm{osc}, N}^{\varepsilon},
$$


where

$$
\varepsilon r_{\mathrm{osc}, N}^{\varepsilon}=\left(R_{\mathrm{osc}, N}^{\varepsilon}+\varepsilon \partial_{t} \widetilde{R}_{\mathrm{osc}, N}^{\varepsilon}\right)+\varepsilon Q^{\varepsilon}\left(\widetilde{R}_{\mathrm{osc}, N}^{\varepsilon}, \varepsilon \widetilde{R}_{\mathrm{osc}, N}^{\varepsilon}+2 v\right) .
$$

We note that the equation satisfied by $\varphi_{N}^{\varepsilon}$ has the advantage that the low-frequency terms have disappeared up to an $\varepsilon$. To show that low-frequency terms are $\mathcal{O}(\varepsilon)$, we recall the following product law (see [3]).

Lemma 3.7. Let $\sigma>2 / 2$ be an integer. A constant $C$ exists such that for all $f \in H^{\sigma}\left(\mathbb{T}^{2}\right)$ and $g \in H^{\sigma+1}\left(\mathbb{T}^{2}\right)$ with $\operatorname{div} f=\operatorname{div} g=0$,

$$
\begin{gathered}
\left|\prec Q^{\varepsilon}(f, g), f \succ_{H^{\sigma}\left(\mathbb{T}^{2}\right)}\right| \leq C\|f\|_{H^{\sigma}\left(\mathbb{T}^{2}\right)}^{2}\|g\|_{H^{\sigma+1}\left(\mathbb{T}^{2}\right)}, \\
\left|\prec Q^{\varepsilon}(f, f), f \succ_{H^{\sigma}\left(\mathbb{T}^{2}\right)}\right| \leq C\|f\|_{H^{\sigma}\left(\mathbb{T}^{2}\right)}^{3} .
\end{gathered}
$$

The following proposition holds.

Proposition 3.8. There exists a constant $C_{N}(T)$ which depends only on $T$ and $N$ such that

$$
\begin{gathered}
\left\|\widetilde{R}_{\mathrm{osc}, N}^{\varepsilon}\right\|_{\mathscr{C}_{0}\left([0, T], H^{s-2}\left(\mathbb{T}^{2}\right)\right) \cap L^{2}\left([0, T], H^{s-1}\left(\mathbb{T}^{2}\right)\right)} \leq C_{N}(T), \\
\left\|r_{\mathrm{osc}, N}^{\varepsilon}\right\|_{L^{2}\left([0, T], H^{s-2}\left(\mathbb{T}^{2}\right)\right)} \leq C_{N}(T) .
\end{gathered}
$$

Proof. Recall that all the functions considered here are truncated in low frequencies. Hence, the result is simply due to the fact that $v \in \mathscr{C}^{0}\left([0, T], H^{s}\left(\mathbb{T}^{2}\right)\right), \partial_{t} v \in \mathscr{C}^{0}([0, T]$, $H^{s-2}\left(\mathbb{T}^{2}\right)$ ) and Lemma 3.7 (see, e.g., [10]).

By the scalar product in $H^{s-2}\left(\mathbb{T}^{2}\right),(3.46)$ gives

$$
\begin{aligned}
& \frac{1}{2} \frac{d}{d t}\left\|\varphi_{N}^{\varepsilon}\right\|_{H^{s-2}\left(\mathbb{T}^{2}\right)}^{2}+2 \prec Q^{\varepsilon}\left(\varphi_{N}^{\varepsilon}, v-\varepsilon \widetilde{R}_{\mathrm{osc}, N}^{\varepsilon}\right), \varphi_{N}^{\varepsilon} \succ_{H^{s-2}\left(\mathbb{T}^{2}\right)} \\
& \quad \leq\left|\prec K^{\varepsilon}-R_{\mathrm{osc}}^{\varepsilon, N}+\varepsilon r_{\mathrm{osc}, N}^{\varepsilon}, \varphi_{N}^{\varepsilon} \succ_{H^{s-2}\left(\mathbb{T}^{2}\right)}\right|+\left|\prec Q^{\varepsilon}\left(\varphi_{N}^{\varepsilon}, \varphi_{N}^{\varepsilon}\right), \varphi_{N}^{\varepsilon} \succ_{H^{s-2}\left(\mathbb{T}^{2}\right)}\right|
\end{aligned}
$$

By Lemma 3.7, we have

$$
\begin{aligned}
\frac{1}{2} \frac{d}{d t}\left\|\varphi_{N}^{\varepsilon}\right\|_{H^{s-2}\left(\mathbb{T}^{2}\right)}^{2} \leq C & {\left[\left\|\varphi_{N}^{\varepsilon}\right\|_{H^{s-2}\left(\mathbb{T}^{2}\right)}^{2}\left(\left\|\varphi_{N}^{\varepsilon}\right\|_{H^{s-2}\left(\mathbb{T}^{2}\right)}+\left\|\varepsilon \widetilde{R}_{\mathrm{osc}, N}^{\varepsilon}-v\right\|_{H^{s-1}\left(\mathbb{T}^{2}\right)}+1\right)\right.} \\
+ & \left.\left\|K^{\varepsilon}-R_{\mathrm{osc}}^{\varepsilon, N}+\varepsilon r_{\mathrm{osc}, N}^{\varepsilon}\right\|_{H^{s-2}\left(\mathbb{T}^{2}\right)}^{2}\right] .
\end{aligned}
$$

Integrating this inequality and using Proposition 3.8, we obtain

$$
\begin{aligned}
\left\|\varphi_{N}^{\varepsilon}(t)\right\|_{H^{s-2}\left(\mathbb{T}^{2}\right)}^{2} \leq & C\left(\left\|K^{\varepsilon}\right\|_{L^{2}\left([0, T], H^{s-2}\left(\mathbb{T}^{2}\right)\right)}+\left\|R_{\mathrm{osc}}^{\varepsilon, N}\right\|_{L^{2}\left([0, T], H^{s-2}\left(\mathbb{T}^{2}\right)\right)}^{2}+\varepsilon^{2} C_{N}^{2}(T)\right) \\
& +C \int_{0}^{t}\left\|\varphi_{N}^{\varepsilon}(\tau)\right\|_{H^{s-2}\left(\mathbb{T}^{2}\right)}^{2}\left(B(T)+\varepsilon C_{N}(T)+\left\|\varphi_{N}^{\varepsilon}(\tau)\right\|_{H^{s-2}\left(\mathbb{T}^{2}\right)}\right) d \tau,
\end{aligned}
$$

where $B(T)=1+\|v\|_{\mathscr{C}_{T}^{0}\left(H^{s-2}\left(\mathbb{T}^{2}\right)\right)}$. 
Put

$$
T^{\star}=\sup \left\{0 \leq t<T /\left\|\varphi_{N}^{\varepsilon}\right\|_{L^{\infty}\left([0, t], H^{s-2}\left(\mathbb{T}^{2}\right)\right)} \leq B(T)\right\} .
$$

Then, for all $0 \leq t<T^{\star}$, we have

$$
\begin{aligned}
\left\|\varphi_{N}^{\varepsilon}(t)\right\|_{H^{s-2}\left(\mathbb{T}^{2}\right)}^{2} \leq & C\left(\left\|K^{\varepsilon}\right\|_{L^{2}\left([0, T], H^{s-2}\left(\mathbb{T}^{2}\right)\right)}+\left\|R_{\mathrm{osc}}^{\varepsilon, N}\right\|_{L^{2}\left([0, T], H^{s-2}\left(\mathbb{T}^{2}\right)\right)}^{2}+\varepsilon^{2} C_{N}^{2}(T)\right) \\
& +C\left(B(T)+\varepsilon C_{N}(T)\right) \int_{0}^{t}\left\|\varphi_{N}^{\varepsilon}(\tau)\right\|_{H^{s-2}\left(\mathbb{T}^{2}\right)}^{2} d \tau .
\end{aligned}
$$

A classical Gronwall estimate gives

$$
\begin{aligned}
\left\|\varphi_{N}^{\varepsilon}(t)\right\|_{H^{s-2}\left(\mathbb{T}^{2}\right)}^{2} \leq & C \exp \left(C T B(T)+C T \varepsilon C_{N}(T)\right) \\
& \times\left(\left\|K^{\varepsilon}\right\|_{L^{2}\left([0, T], H^{s-2}\left(\mathbb{T}^{2}\right)\right)}+\left\|R_{\mathrm{osc}}^{\varepsilon, N}\right\|_{L^{2}\left([0, T], H^{s-2}\left(\mathbb{T}^{2}\right)\right)}^{2}+\varepsilon^{2} C_{N}^{2}(T)\right) .
\end{aligned}
$$

For $N$ large and $\varepsilon$ small enough, Proposition 3.6 leads to

$$
\left\|\varphi_{N}^{\varepsilon}(t)\right\|_{H^{s-2}\left(\mathbb{T}^{2}\right)} \leq \frac{B(T)}{2},
$$

which implies that $T^{\star}=T$.

Using Proposition 3.6 and letting $\varepsilon \rightarrow 0, N \rightarrow+\infty$, we get

$$
W^{\varepsilon} \longrightarrow 0 \quad \text { in } \mathscr{C}^{0}\left([0, T], H^{s-2}\left(\mathbb{T}^{2}\right)\right)
$$

An interpolation argument completes the proof.

Remark 3.9. For the particular choice $k=(6 p, 0,3 p)$ and $m=(4 p, 0,-8 p), p \in \mathbb{Z}^{*}$, a direct computation gives

$$
\omega_{n, k, m}=\sigma_{1} \frac{n_{3}}{|n|}-\sigma_{2} \frac{k_{3}}{|k|}-\sigma_{3} \frac{m_{3}}{|m|}=0
$$

Then, the resonance set is not empty and the unit torus $\mathbb{T}^{2}$ is a resonant one.

\section{Case of the whole space $\mathbb{R}^{3}$}

Theorem 1.4 and its proof are similar to those given in [2]. We give them here for the sake of completeness.

4.1. Proof of Theorem 1.4. Let us designate by $\mu$ the Lebesgue measure on $\mathbb{R}^{3}$. Since

$$
\mu\left(\left\{\xi \in \mathbb{R}^{3}, \xi_{2}=0\right\}\right)=0,
$$


then one infers that for any function $f$,

$$
\|f\|_{H^{\sigma}\left(\mathbb{R}^{3}\right)}^{2}=\int_{\mathbb{R}^{3}}\left(1+|\xi|^{2}\right)^{\sigma}|\hat{f}(\xi)|^{2} d \xi=\int_{\xi_{2} \neq 0}\left(1+|\xi|^{2}\right)^{\sigma}|\hat{f}(\xi)|^{2} d \xi
$$

Consequently, it suffices to estimate $\left|\widehat{u^{\varepsilon}}(t, \xi)\right|$ for $\xi_{2} \neq 0$.

Observe that the same computation used in the periodic case still holds in the case of the whole space, just change $k$ by $\xi$. Precisely, as in the periodic case, one just have to change $k$ by $\xi$, apply the Duhamel formula and the Cauchy-Schwarz inequality to obtain, for $\xi_{3} \neq 0$,

$$
\left|\widehat{U^{\varepsilon}}(t, \xi)\right|^{2} \leq 2 \exp \left(-\frac{2 t}{\varepsilon} \omega(\xi)\right)\left|\widehat{U_{0}}(\xi)\right|^{2}+\varepsilon\left(\frac{1-\exp (-(T / \varepsilon) \omega(\xi))}{\omega(\xi)}\right) \int_{0}^{T}\left|\widehat{F^{\varepsilon}}(\tau, \xi)\right|^{2} d \tau
$$

It easily follows that

$$
\begin{aligned}
\left\|U^{\varepsilon}(t)\right\|_{H^{s-1}}^{2} \leq & 2 \int_{\mathbb{R}^{3}}\left(1+|\xi|^{2}\right)^{s-1}\left|\widehat{U_{0}}(\xi)\right|^{2} \exp \left(-\frac{2 t}{\varepsilon} \omega(\xi)\right) d \xi \\
& +2 \int_{\mathbb{R}^{3}} \int_{0}^{t} \frac{\varepsilon}{2 \omega(\xi)}\left(1-\exp \left(-\frac{2 t}{\varepsilon} \omega(\xi)\right)\right)\left|\widehat{F^{\varepsilon}}(\tau, \xi)\right|^{2}\left(1+|\xi|^{2}\right)^{s-1} d \xi \\
\leq & 2 \int_{\mathbb{R}^{3}}\left(1+|\xi|^{2}\right)^{s-1}\left|\widehat{U_{0}}(\xi)\right|^{2} \exp \left(-\frac{2 t}{\varepsilon} \omega(\xi)\right) d \xi+T \int_{0}^{t}\left\|\widehat{F^{\varepsilon}}(\tau)\right\|_{H^{s-1}}^{2} d \tau
\end{aligned}
$$

where

$$
F^{\varepsilon}:=\left(\varepsilon \Delta u^{\varepsilon}-\mathbb{P}\left(u^{\varepsilon} \cdot \nabla u^{\varepsilon}\right)+\mathbb{P}\left(b^{\varepsilon} \cdot \nabla b^{\varepsilon}\right) ; b^{\varepsilon} \cdot \nabla u^{\varepsilon}-u^{\varepsilon} \cdot \nabla b^{\varepsilon}\right) .
$$

Using the energy estimate (1.8) and product laws, we get

$$
\begin{gathered}
\int_{0}^{t}\left\|\varepsilon \Delta u^{\varepsilon}(\tau)\right\|_{H^{s-1}}^{2} \leq \varepsilon \cdot \varepsilon \int_{0}^{t}\left\|\nabla u^{\varepsilon}\right\|_{H^{s-1}}^{2} \leq C \varepsilon \\
\int_{0}^{t}\left\|u^{\varepsilon} \cdot \nabla u^{\varepsilon}(\tau)\right\|_{H^{s-1}}^{2} d \tau \leq C \int_{0}^{t}\left\|u^{\varepsilon}(\tau)\right\|_{H^{s-1}}^{2} d \tau \\
\int_{0}^{t}\left\|b^{\varepsilon} \cdot \nabla b^{\varepsilon}(\tau)\right\|_{H^{s-1}}^{2} d \tau \leq C \int_{0}^{t}\left\|\nabla b^{\varepsilon}(\tau)\right\|_{H^{s}}^{2} d \tau \leq C \varepsilon \\
\int_{0}^{t}\left\|u^{\varepsilon} \cdot \nabla b^{\varepsilon}(\tau)\right\|_{H^{s-1}}^{2} d \tau \leq C \int_{0}^{t}\left\|\nabla b^{\varepsilon}(\tau)\right\|_{H^{s}}^{2} d \tau \leq C \varepsilon \\
\int_{0}^{t}\left\|b^{\varepsilon} \cdot \nabla u^{\varepsilon}(\tau)\right\|_{H^{s-1}}^{2} d \tau \leq C \int_{0}^{t}\left\|b^{\varepsilon}(\tau)\right\|_{H^{s-1}}^{2} d \tau .
\end{gathered}
$$


Consequently, one infers that

$$
\begin{aligned}
\left\|U^{\varepsilon}(t)\right\|_{H^{s-1}}^{2} \leq & 2 \int_{\mathbb{R}^{3}}\left(1+|\xi|^{2}\right)^{s-1}\left|\widehat{U}_{0}(\xi)\right|^{2} \exp \left(-\frac{2 t}{\varepsilon} \omega(\xi)\right) d \xi \\
& +C \varepsilon T+C \int_{0}^{t}\left\|U^{\varepsilon}(\tau)\right\|_{H^{s-1}}^{2} d \tau .
\end{aligned}
$$

Using Gronwall lemma, the Lebesgue theorem, and interpolation argument, we achieve the proof.

Remark 4.1. Since the limit system $(\mathscr{L} \mathscr{S})$ is the two-dimensional Euler equation, then, in the case of the whole space, a refined convergence result can be obtained for initial data with $2 \mathrm{D}$ and $3 \mathrm{D}$ components. To do so, we can proceed as in [6] and approximate the solution $\left(u^{\varepsilon}, b^{\varepsilon}\right)$ of the MHD system by the sum of the two-dimensional Navier-Stokes solution and the MHD linear part solution. For explicit computation, we refer the reader to [2].

\section{References}

[1] A. Babin, A. Mahalov, and B. Nicolaenko, "Global splitting, integrability and regularity of 3D Euler and Navier-Stokes equations for uniformly rotating fluids," European Journal of Mechanics. B Fluids, vol. 15, no. 3, pp. 291-300, 1996.

[2] J. Benameur, M. Ghazel, and M. Majdoub, "About MHD system with small parameter," Asymptotic Analysis, vol. 41, no. 1, pp. 1-21, 2005.

[3] J. Benameur, S. Ibrahim, and M. Majdoub, "Asymptotic study of a magneto-hydrodynamic system," Differential and Integral Equations, vol. 18, no. 3, pp. 299-324, 2005.

[4] J.-Y. Chemin, "About Navier-Stokes Equations," Publication du Laboratoire Jacques-Louis Lions, Université de Paris VI, R96023, 1996.

[5] J.-Y. Chemin, B. Desjardins, I. Gallagher, and E. Grenier, "Fluids with anisotropic viscosity," M2AN. Mathematical Modelling and Numerical Analysis, vol. 34, no. 2, pp. 315-335, 2000.

[6] J.-Y. Chemin, B. Desjardins, I. Gallagher, and E. Grenier, "Anisotropy and dispersion in rotating fluids," in Nonlinear Partial Differential Equations and Their Applications. Collège de France Seminar, Vol. XIV (Paris, 1997/1998), vol. 31 of Studies in Mathematics and Its Applications, pp. 171-192, North-Holland, Amsterdam, 2002.

[7] B. Desjardins, E. Dormy, and E. Grenier, "Stability of mixed Ekman-Hartmann boundary layers," Nonlinearity, vol. 12, no. 2, pp. 181-199, 1999.

[8] E. Dormy, "Modélisation numérique de la dynamo terrestre," Thèse de Doctorat, Institut de Physique du Globe de Paris, Paris, 1997.

[9] I. Gallagher, "Applications of Schochet's methods to parabolic equations," Journal de Mathématiques Pures et Appliquées. Neuvième Série, vol. 77, no. 10, pp. 989-1054, 1998.

[10] I. Gallagher, "Asymptotic of the solutions of hyperbolic equations with a skew-symmetric perturbation," Journal of Differential Equations, vol. 150, no. 2, pp. 363-384, 1998.

[11] E. Grenier, "Oscillatory perturbations of the Navier-Stokes equations," Journal de Mathématiques Pures et Appliquées. Neuvième Série, vol. 76, no. 6, pp. 477-498, 1997.

[12] J.-L. Joly, G. Métivier, and J. Rauch, "Generic rigorous asymptotic expansions for weakly nonlinear multidimensional oscillatory waves,” Duke Mathematical Journal, vol. 70, no. 2, pp. 373-404, 1993. 
[13] J.-L. Joly, G. Métivier, and J. Rauch, "Coherent and focusing multidimensional nonlinear geometric optics," Annales Scientifiques de l'École Normale Supérieure. Quatrième Série, vol. 28, no. 1, pp. 51-113, 1995.

[14] S. Klainerman and A. Majda, "Singular limits of quasilinear hyperbolic systems with large parameters and the incompressible limit of compressible fluids," Communications on Pure and Applied Mathematics, vol. 34, no. 4, pp. 481-524, 1981.

[15] J.-L. Lions, "Quelques méthodes de résolution des problèmes aux limites non linéaires," Dunod, Paris, 1969.

[16] A. Majda, Compressible Fluid Flow and Systems of Conservation Laws in Several Space Variables, vol. 53 of Applied Mathematical Sciences, Springer, New York, 1984.

[17] M.-G. Paicu, "Étude asymptotique pour les fluides anisotropes en rotation rapide dans le cas périodique," Journal de Mathématiques Pures et Appliquées. Neuvième Série, vol. 83, no. 2, pp. 163-242, 2004.

[18] S. Schochet, "The compressible Euler equations in a bounded domain: existence of solutions and the incompressible limit," Communications in Mathematical Physics, vol. 104, no. 1, pp. 49-75, 1986.

[19] S. Schochet, "Fast singular limits of hyperbolic PDEs," Journal of Differential Equations, vol. 114, no. 2, pp. 476-512, 1994.

[20] R. Temam, Navier-Stokes Equations, vol. 2 of Studies in Mathematics and Its Applications, NorthHolland, Amsterdam, 1984.

Ridha Selmi: Département de Mathématiques, Institut Supérieur d’Informatique, Université de Tunis El-Manar, 2 rue Abou Rayhane Bayrouni, 2080 l'Ariana, Tunisia;

Laboratoire des Equations aux Derivées Partielles et Applications,

Faculté des Sciences de Tunis, Compus Universitaire 2092, Tunis, Tunisia

E-mail address: ridha.selmi@isi.rnu.tn 


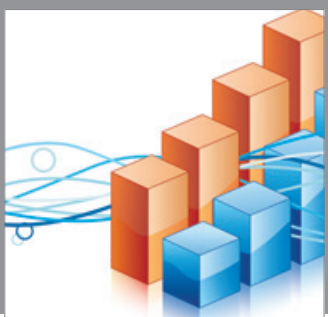

Advances in

Operations Research

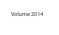

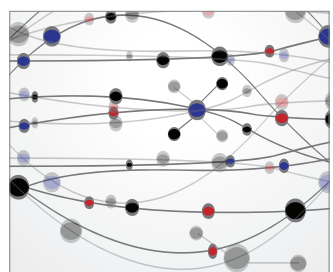

\section{The Scientific} World Journal
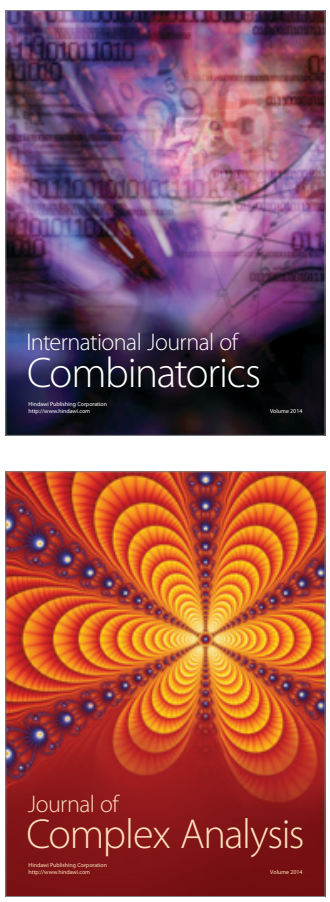

International Journal of

Mathematics and

Mathematical

Sciences
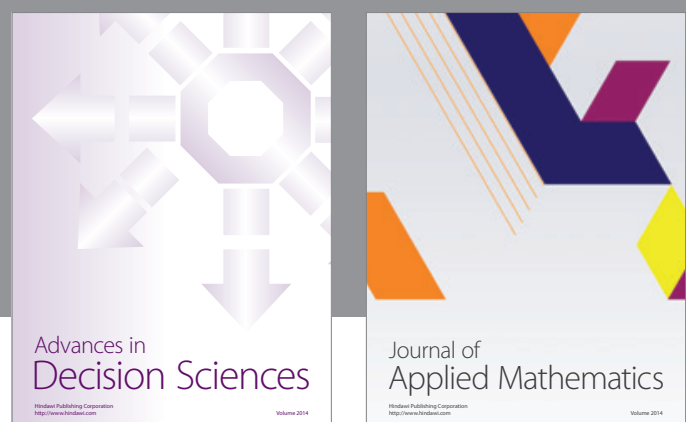

Journal of

Applied Mathematics
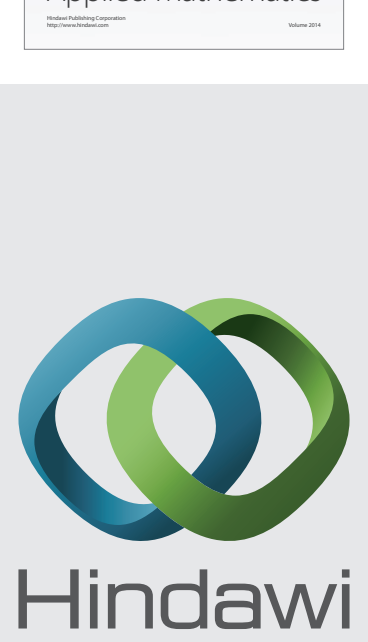

Submit your manuscripts at http://www.hindawi.com
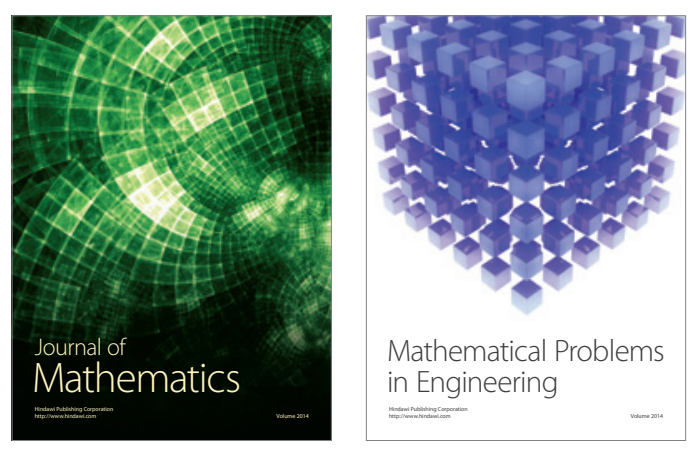

Mathematical Problems in Engineering
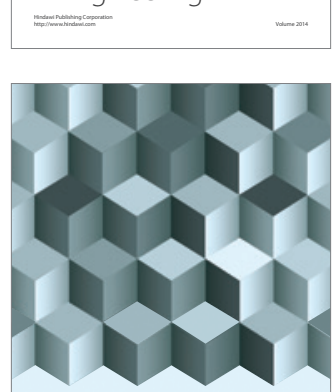

Journal of

Function Spaces
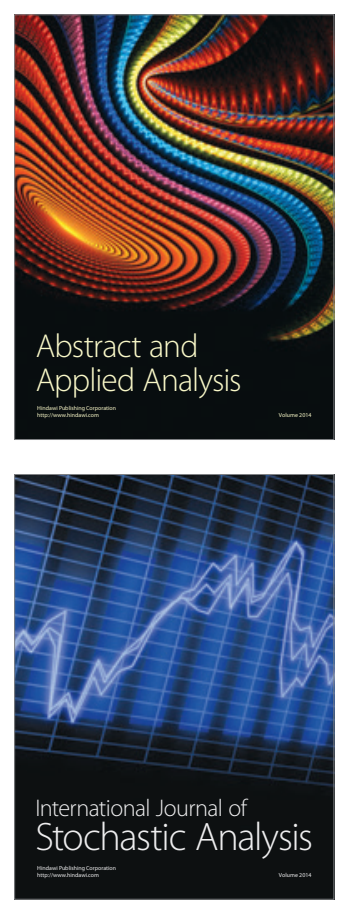

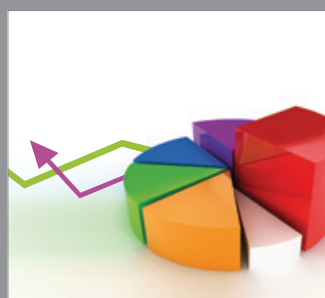

ournal of

Probability and Statistics

Promensencen
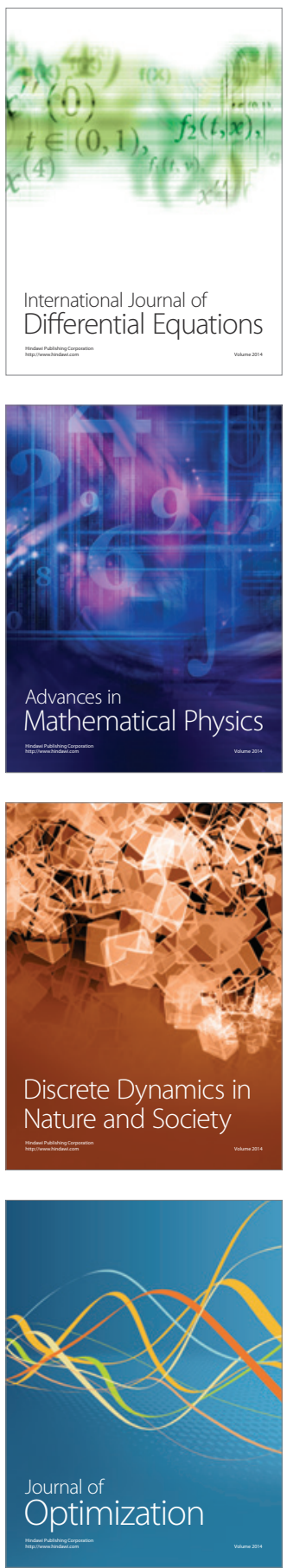